\title{
The Effect of Long-Range Interactions on Development of Thermal Waves in the Radiation-Cooling Dispersed Flow
}

\author{
A. A. Safronov, A. A. Koroteev, N. I. Filatov, A. L. Grigoriev
}

The influence of long-range interactions on the progress of heat waves in the radiationcooling disperse flow is considered. It is shown that the system exhibits oscillations attendant on the process of establishing an equilibrium temperature profile. The oscillation amplitude and the rate of oscillation damping are determined. The conditions under which the radiation cooling process can be unstable with respect to temperature field perturbations are revealed. The results of theoretical analysis and numerical calculation of the actual droplet flow are compared.

Keywords: disperse flows, radiative heat transfer, long-range interactions, instability

Received May 17, 2018

Accepted July 16, 2018

This work was supported by the Russian Foundation for Basic Research (Grant no. 16-08-01050).

Andrey A. Safronov

a.a.safr@yandex.ru

Nikolay I. Filatov

feel_57@mail.ru

Alexey L. Grigoriev

grigorev@kerc.msk.ru

Keldysh Research Center

ul. Onezhskaya 8, Moscow, 125438, Russia

Anatoly A. Koroteev

chkt@yandex.ru

Moscow Aviation Institute (National Research University)

Volokolamskoe sh. 4, Moscow, 125993, Russia 


\section{Introduction}

Solving new scientific and engineering problems, as well as problems in the sphere of information and telecommunication, related to the use of space, calls for a significant increase in spacecraft power-to-weight ratio. The low-grade heat rejection system becomes the most problematic constituent of power systems. When power is increased considerably, conventional panel radiators have a too large area of surface, mass and meteorite vulnerability. Calculations show that the limiting power of long-term functioning spacecrafts with panel radiators does not exceed 100 to $200 \mathrm{~kW}$. With the droplet radiator (LDR) in place it becomes possible to make megawatt-class power spacecrafts [1]. Figure 1 shows a schematic diagram of the LDR. The generator produces droplet flow, cooling down with its propagation in space toward the hydrocollector catching it.

The problem of the radiation cooling of the droplet veil was studied earlier in relation to radiators with particle size from 5 to $30 \mu \mathrm{m}$, whose arrangement was considered to be uniform $[2,3]$. It was supposed that the droplet temperature is $700 \mathrm{~K}[4]$. The analysis of the energy characteristics was based on the model of an isotropic-diffusing grey medium. For determination of the temperature field in the flow, equations of radiation diffusion transport were solved; it was believed that there are no long-range radiation interactions in the system. The droplet flow cooling process was considered to be a propagation of the heat wave in a medium with nonlinear thermal conductivity [3]. The use of low-grade radiators makes it possible to increase the efficiency of the spacecraft power system and to decrease evaporation losses of the working fluid. The droplet radius in this DR is $r \sim 100 \mu \mathrm{m}$ and is determined by technical feasibilities of substance dispersing [5]. As the temperature is low enough $(T \sim 400 \mathrm{~K})$, the thermal emission spectrum is concentrated in the region of wavelengths at which the working fluid efficiently absorbs the emission. The integral emissivity of droplets approaches unity. Calculations reveal that the use of the structured droplet flow (Fig. 2) enables an increase in the LDR power by a few tens of per cent as compared to the case of uniform arrangement of particles [6].

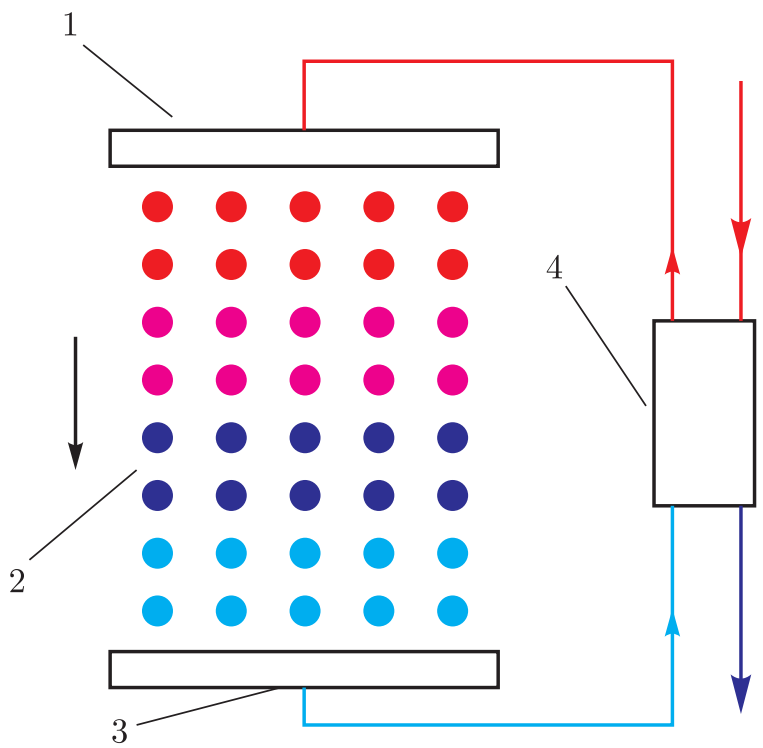

Fig. 1. A schematic diagram of the droplet cooler radiator. 1 - the droplet flow generator; $2-$ the droplet flow; 3 - the hydrocollector; 4 - the heat exchanger; 5 - the power system loop. 


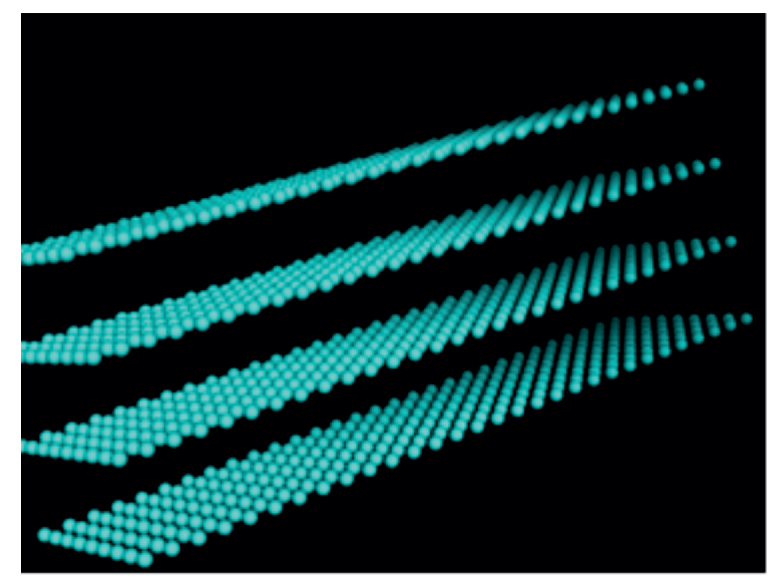

Fig. 2. The structured droplet flow involving the four droplet layers.

Long-range radiation interactions make a noticeable contribution to overradiation of particles. The availability of such interactions in the medium essentially changes the structure of propagating heat waves [7]. This work investigates the influence of long-range interactions on the progress of heat waves in the radiation-cooling disperse flow of the low-grade LDR.

\section{The mathematical model}

The structured droplet flow involving several elements - parallel thin droplet layers - is considered (Fig. 2). The distance between structure elements is thought to exceed the layer thickness, all the droplets in the flow are identical. For identification of the particles the index $i$ is used. In terms of mutual overradiation of the droplets the particle cooling equation is written in the form

$$
\left(\frac{\partial T_{i}}{\partial t}+u \frac{\partial T_{i}}{\partial x}\right) m c=-4 \pi r^{2} \varepsilon(r, T) \sigma T_{i}^{4}(t, x)+Q_{i \Sigma}(t, x)+P_{\odot i},
$$

where $t$ and $x$ are the time and the coordinate, $m, u$ are the mass and the rate of the droplets, $T_{i}$ is the temperature of the $i$-th droplet, $c$ is the substance density, $\sigma$ is the Stefan-Boltzmann constant, $P_{\odot i}$ is the droplet heating power due to external radiation, $\varepsilon$ is the efficient emissivity of the droplet that is assumed to be constant. The term $Q_{i \Sigma}$ characterizes the radiation interaction of the droplet with other particles of the flow. A little variation in the temperature through the droplet volume is not taken into account in Eq. (1.1).

In the structured droplet flow the conditions of particles cooling in each layer are identical. This work has to do with the cooling process of droplet layers, and so the index $i$ defines the structure element number (Fig. 3), the overradiation of the droplet with the veil can be written as

$$
Q_{i \Sigma}(t, x)=\varepsilon \sum_{j=1}^{N} \int_{-x}^{l-x} f_{i, j}(\widetilde{x}) T_{i}^{4}(t, x+\widetilde{x}) d \widetilde{x},
$$

where $l$ is the length of the droplet flight in the veil, $f_{i, j}$ is the function of the emissivity distribution between the droplet layers with the $i$ and $j$ numbers. The physical meaning of the integral is the summation of overradiation energies of all veil droplets with a droplet at hand.

RUSSIAN JOURNAL OF NONLINEAR DYNAMICS, 2018, 14(3), 343-354 


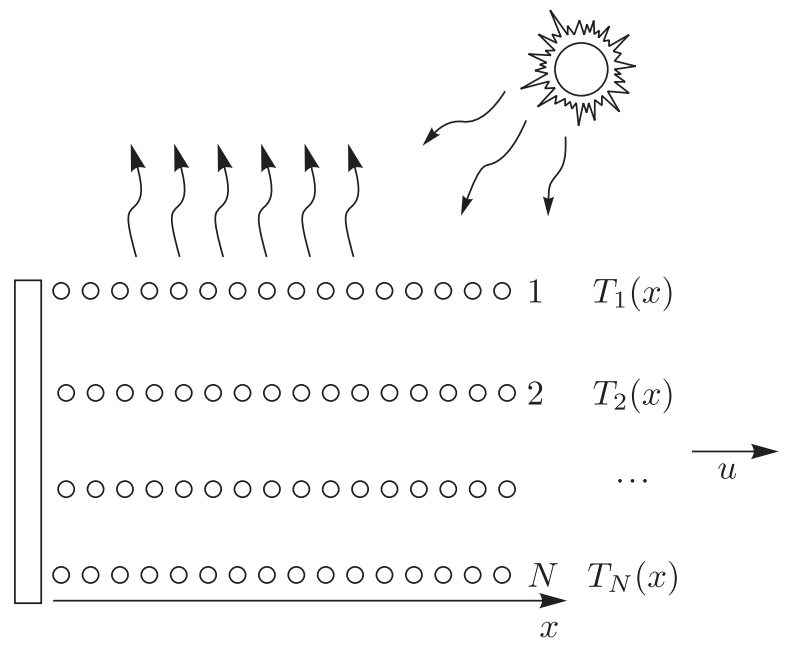

Fig. 3. Cooling of the structured droplet flow.

As the distance between the droplet layers is much larger than their thickness, it is assumed that for the emissivity distribution function the following equality is satisfied: $f_{x, i, j}(x)=$ $=f_{|i-y|}(x)$. In view of this relationship and using the notation $\alpha=3 \sigma \varepsilon /($ rcu $\rho)$, where $\rho$ is the substance density, Eq. (1.1) may be written in the form

$$
\frac{1}{\alpha u} \partial_{t} T_{i}+\frac{1}{\alpha} \partial_{x} T_{i}=-T_{i}^{4}+\varepsilon \sum_{j=1}^{N} \int_{-x}^{l-x} T_{j}^{4}(\widetilde{x}+x) f_{|i-j|}(\widetilde{x}) d \widetilde{x}+\frac{P_{\odot i}}{m c u} .
$$

The integro-differential system of equations (1.3) can be represented as a delayed system. The magnitude of time lag $\Delta x_{i, j}$, defined by the relationship:

$$
\varphi_{|i-j|} T_{i}^{4}\left(x-\Delta x_{i, j}\right)=\int_{-x}^{l-x} T_{i}^{4}(\widetilde{x}+x) f_{|i-j|}(\widetilde{x}) d \widetilde{x}
$$

where $\varphi_{|i-j|}=\int_{0}^{l} f_{|i-j|}(\widetilde{x}) d \widetilde{x}$, is introduced. Neglecting the third derivative of the temperature with respect to coordinate, the magnitude of the time lag is equal to

$$
\Delta x_{i, j}(t, x)=-\frac{1}{\partial_{x} T_{j}}\left(T_{j} \partial_{x} T_{j}+3\left(\partial_{x} T_{j}\right)^{2}\right) \int_{-l / 2}^{l / 2} f_{|i-j|}(\widetilde{x}) \widetilde{x}^{2} d \widetilde{x} .
$$

Time-steady solutions of Eq. (1.3) are considered. The dynamical system is solved on a family of characteristics $x^{\prime}=u t-x=$ const. For compactness of the notation, the prime of the magnitude $x^{\prime}$ is dropped. Then the cooling equation is written in the form

$$
\frac{d T_{i}}{d x}=-\alpha T_{i}^{4}(x)+\varepsilon \alpha \sum_{j=1}^{N} \varphi_{|i-j|} T_{j}^{4}\left(x-\Delta x_{|i-j|}\right)+\frac{\alpha P_{\odot i}}{m c u} .
$$

The initial condition for this system of equations is the temperature field in the droplet flow at a certain instant of time. 


\section{The idealized problem of establishing a temperature profile in the radiation cooling disperse flow}

The droplet cooling process occurs as follows. At first the temperature of all particles is identical and equals $T_{0}$. Then the temperature of the droplets at periphery proves to be less than in the core of the flow (Fig. 4). At a certain distance from the droplet generator, the equilibrium profile of the flow temperature is established. Then, as the cooling goes on, the profile remains unchanged. In what follows, the process of establishing the equilibrium temperature profile is considered.
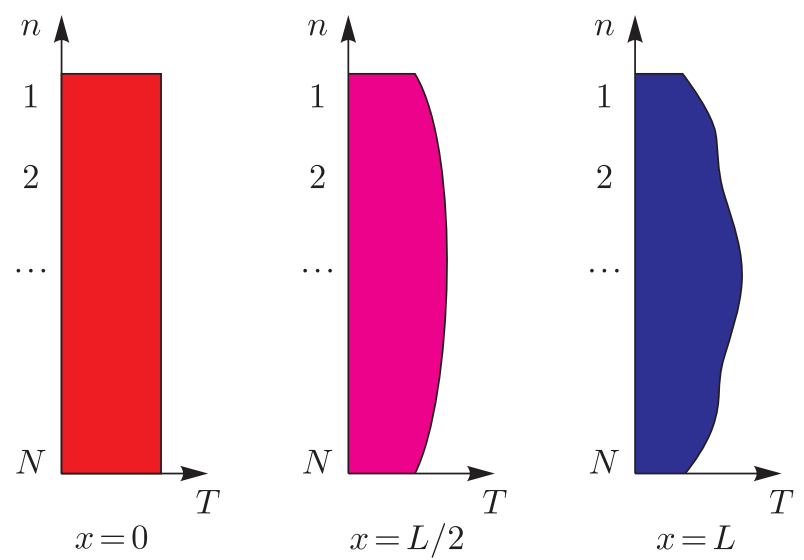

Fig. 4. The process of establishing the droplet flow temperature profile.

External radiation is assumed to be absent, and the magnitude $\Delta x_{i, j}$ is negligible for all values of $i$ and $j$. In this case the system (1.6) takes the form

$$
\frac{d T_{i}}{d x}=-\alpha T_{i}^{4}(x)+\varepsilon \alpha \sum_{j=1}^{N} \varphi_{|i-j|} T_{j}^{4}(x) .
$$

The system (2.1) was investigated in [8]. For its solution, the following change of variables is made:

$$
\begin{aligned}
\xi & =\left(1+3 \alpha T_{0}^{3} x\right)^{-1 / 12}, \\
T_{i}(\xi) & =T_{0} \xi^{4} \tau_{i}(\xi) .
\end{aligned}
$$

Physically, the magnitude $\xi$ is the cooling rate of a freely cooling droplet ( $\xi$ decreases as the coordinate increases), the dimensionless temperature $\tau$ is the ratio of the temperature of the droplet cooling down in the LDR flow and that of the freely cooling droplet. The system (2.1) in the new variables becomes

$$
4 \xi \frac{d \tau_{i}}{d \xi}=\tau_{i}^{4}-\tau_{i}-\varepsilon \sum_{j=1}^{N} \varphi_{|i-j|} \tau_{j}^{4} .
$$

The initial condition is $\tau_{i}=1$.

The system (2.3) describes the process of establishing the droplet flow temperature profile. In this work its solution is found for the axially symmetric droplet flow involving the heated core 


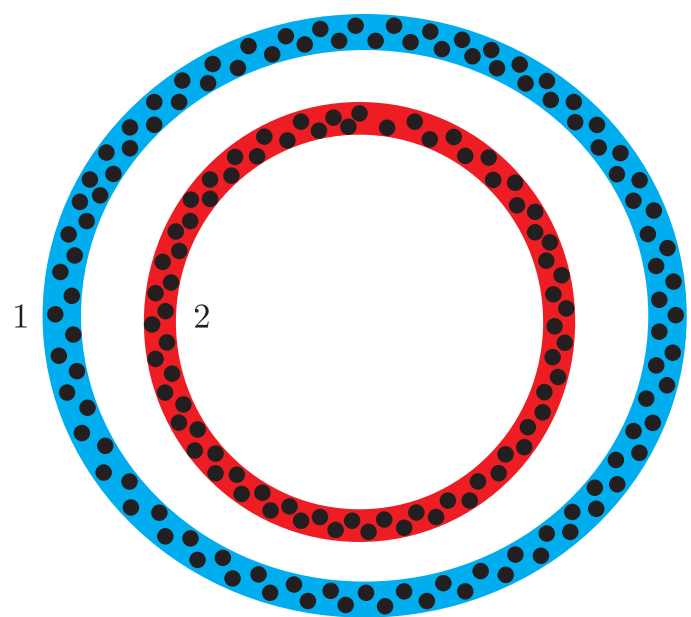

Fig. 5. The model of the droplet flow consisting of the periphery (1) and the core (2).

and the cooled periphery (Fig. 5). At the center of the flow the temperature field $\tau_{c}$ changes slowly, and at the periphery, $\tau_{p}$, it changes much faster. The cooling equations are

$$
\begin{aligned}
& \frac{1}{4} \xi \frac{d \tau_{c}}{d \xi}=\left(1-\varphi_{1}\right) \tau_{c}{ }^{4}-\tau_{c}-\varepsilon \psi_{1} \tau_{p}{ }^{4} \\
& \frac{1}{4} \xi \frac{d \tau_{p}}{d \xi}=\left(1-\varphi_{2}\right) \tau_{p}{ }^{4}-\tau_{p}-\varepsilon \psi_{2} \tau_{c}{ }^{4}
\end{aligned}
$$

where $\psi_{1}$ and $\psi_{2}$ are the angular emissivities of the core of the flow to periphery and backwards, respectively, $\varphi_{1}$ and $\varphi_{2}$ are the coefficients of self-radiation of the core and the periphery.

The dissipative system (2.4) has a single equilibrium position $\left(\tau_{c}^{*} ; \tau_{p}^{*}\right)$ describing the established temperature profile. To investigate the solution in a small vicinity of the equilibrium position, we introduce local variables $\delta x$ and $\delta y: \tau_{c}=\tau_{c}^{*}+\delta x$ and $\tau_{p}=\tau_{p}^{*}+\delta y$. The linear approximation of the system (2.4) is

$$
4 \xi \frac{d}{d \xi} \boldsymbol{\delta} \tau=\widehat{A} \boldsymbol{\delta} \tau
$$

where the matrix $\widehat{A}=\left(\begin{array}{cc}4 \tau_{c}^{* 3}\left(1-\varphi_{1}\right)-1 & -4 \varepsilon \psi_{1} \tau_{p}{ }^{* 3} \\ -4 \varepsilon \psi_{2} \tau_{c}{ }^{* 3} & 4 \tau_{p}^{* 3}\left(1-\varphi_{2}\right)-1\end{array}\right)$, and the vector $\boldsymbol{\delta} \boldsymbol{\tau}=\left(\begin{array}{c}\delta x \\ \delta y\end{array}\right)$. The system (2.5) may be generalized to the case of larger dimensionality.

In [8] it has been revealed that the equilibrium position in the system (2.5) is a stable node (Fig. 6), and in the general case, the process of establishing the droplet flow temperature profile is not monotonic and may involve oscillations.

For modeling of the effect of the external radiation on the process of establishing the temperature profile, the term $\Psi_{i}(\xi)$ describing the action of the factor is added in Eq. (2.1)

$$
4 \xi \frac{d \tau_{i}}{d \xi}=\tau_{i}^{4}-\tau_{i}-\varepsilon \sum_{j=1}^{N} \varphi_{|i-j|} \tau_{j}^{4}-\Psi_{i}(\xi) .
$$




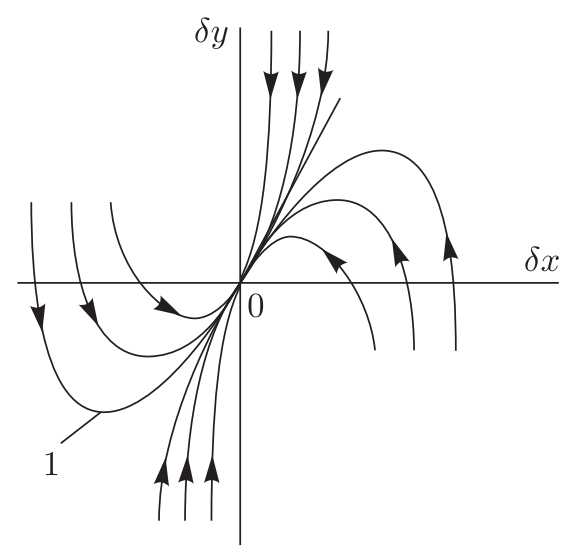

Fig. 6. Solutions of the system (2.5) near the singular point. The system trajectory with nonmonotonic variation in the dimensionless temperature is denoted by the number 1 .

In a neighborhood of the equilibrium position of the unperturbed problem (2.3) the trajectory of the system (2.6) can be described by the equation

$$
4 \xi \frac{d}{d \xi} \boldsymbol{\delta} \tau=\widehat{A} \boldsymbol{\delta} \tau-\boldsymbol{\Psi}(\xi)
$$

The equilibrium position of the system (2.7) moves at a rate $\partial_{\xi} \boldsymbol{\delta} \boldsymbol{r}$ :

$$
\partial_{\xi} \boldsymbol{\delta} \boldsymbol{r}(\xi)=\widehat{A}^{-1} \frac{d}{d \xi} \boldsymbol{\Psi}(\xi)
$$

where $\widehat{A}^{-1}$ is the matrix inverse to the matrix $\widehat{A}$. The system's trajectory follows the equilibrium position at a certain distance $\Delta$ :

$$
\Delta=\frac{1}{\lambda_{2}}\left|\partial_{\xi} \boldsymbol{\delta} \boldsymbol{r}(\xi)\right|
$$

From the relation (2.9) it is seen that in the general case the velocity vector of the motion of the equilibrium position $\partial \xi \delta \boldsymbol{r}$ can change its direction. As a result of this and the fact that the phase trajectories have a rather complex form (Fig. 6), oscillations may arise in the solution of the system (2.6). Their amplitude is estimated by the magnitude $\Delta$.

\section{The effect of long-range radiation interactions on the process of establishing the temperature profile}

The analysis of the solution of the set of equations (1.6) is carried out with the dimensionless variables $\xi$ and $\tau, \Delta x i, j$ is assumed to be a small magnitude. The linear approximation of the system (1.6) is written as

$$
4 \xi \frac{d \tau_{i}}{d \xi}=\tau_{i}^{4}-\tau_{i}-\varepsilon \sum_{j=1}^{N} \varphi_{|i-j|}\left[1+4 \alpha T_{0}^{3} \xi^{12} \Delta x_{|i-j|}\right] \tau_{j}^{4}\left(\xi+\Delta x_{|i-j|} \frac{\alpha T_{0}^{3} \xi^{12}}{4}\right)-\Psi_{i} .
$$

Due to the fact that $\xi$ diminishes with increasing coordinate, the delayed system (1.6) in new variables is transformed into an advanced system (3.1). To simplify the problem, it is assumed

RUSSIAN JOURNAL OF NONLINEAR DYNAMICS, 2018, 14(3), 343-354 
that the droplet flow involves the two structure elements - the core and the periphery (Fig. 5), the external radiation is absent, $\varepsilon=1$, the magnitude of the time lag is identical for all the structure elements and equals $\Delta x$. The notation $w=\alpha T_{0}^{3} \xi^{12} \Delta x$ is introduced. By analogy with the previous section, the solution of the problem is analyzed in a neighborhood of the equilibrium position, and a change to the variables $\delta x$ and $\delta y$ is made. As a result, the system (3.1) is written in the form

$$
4 \xi \frac{d}{d \xi} \boldsymbol{\delta} \tau=\widehat{B} \boldsymbol{\delta} \tau+\widehat{\Omega} \boldsymbol{\delta} \tau(\xi+w / 4)
$$

where the matrix $\widehat{B}=\left(\begin{array}{cc}4 \tau_{c}^{* 3}\left(1-\varphi_{1}\right)-1 & 0 \\ 0 & 4 \tau_{p}^{* 3}\left(1-\varphi_{2}\right)-1\end{array}\right)$, the matrix $\widehat{\Omega}=\left(\begin{array}{cc}0 & -4 \psi_{1} \tau_{p}^{* 3}(1+4 w) \\ -4 \psi_{2} \tau_{c}^{* 3}(1+4 w) & 0\end{array}\right)$.

The equilibrium position of the system (3.2) is in motion with a variation in the magnitude $\xi$ at the velocity

$$
\partial_{\xi} \boldsymbol{\delta} \boldsymbol{r}(\xi) \approx-3 w\left(\widehat{A}^{-1}\left(\begin{array}{c}
\psi_{1} \tau_{p}^{* 3} \\
\psi_{2} \tau_{c}^{* 3}
\end{array}\right)\right) .
$$

Since the velocity vector of the motion of the equilibrium position $\partial \xi \delta \boldsymbol{r}$ changes its direction with the varying $\xi$, oscillations of the system's phase trajectory can arise. Their amplitude is estimated by the formula (2.9).

Perturbation development in the disperse flow temperature field was modeled using the characteristic equation for the advanced system (3.2):

$$
\operatorname{det}\left(\lambda \widehat{E}-\widehat{B}-\widehat{\Omega} e^{\lambda w / 4}\right)=0
$$

Since $\xi$ decreases with increasing coordinate, the growing perturbations correspond to a negative value of $\lambda$. Using the notation $a=\left(4\left(1-\varphi_{1}\right) \tau_{c}^{* 3}+4\left(1-\varphi_{2}\right) \tau_{p}^{* 3}-2\right), b=\left(1-4\left(1-\varphi_{1}\right) \tau_{c}^{* 3}\right)(1-$ $\left.-4\left(1-\varphi_{2}\right) \tau_{p}^{* 3}\right)$ and $d=16 \psi_{1} \psi_{2} \tau_{c}^{* 3} \tau_{p}^{* 3}$, the relation (3.4) may be rewritten as

$$
\lambda^{2}-a \lambda+b=d(1+4 w)^{2} e^{\lambda w / 2} .
$$

Depending on the parameter values of the problem, (3.5) may have one, two or three solutions. For values of the parameters $\psi_{1}, \psi_{2}, \varphi_{1}$ and $\varphi_{2}$, which have a physical meaning, there are solutions of the system (3.5) that are relevant to growing perturbations of the temperature field in the disperse flow. So, when $\psi_{1}=0.3, \psi_{2}=0.12, \varphi_{1}=0.85$ and $\varphi_{2}=0.3$, with $w>0.14$, Eq. (3.5) has one negative solution (Fig. 7). When the time lag in the system is small and $w<0.14$, the temperature field perturbations damp out.

In addition, the problem of determination of the minimum value of the time lag, $\Delta x_{\min }$, at which for the initial particle temperature $T_{0}$ the radiation cooling process is unsteady relative to the temperature field perturbations, has been solved. The magnitude $w$ may be represented in the form

$$
w(x)=\alpha T_{0}^{3} \Delta x\left(1+3 \alpha T_{0}^{3} x\right)^{-1} .
$$

From the analysis of Eq. (3.6) it follows that $w$ diminishes with increasing coordinate and has the largest value, close to the generator, where the value of $x$ nears zero. By virtue of the fact 


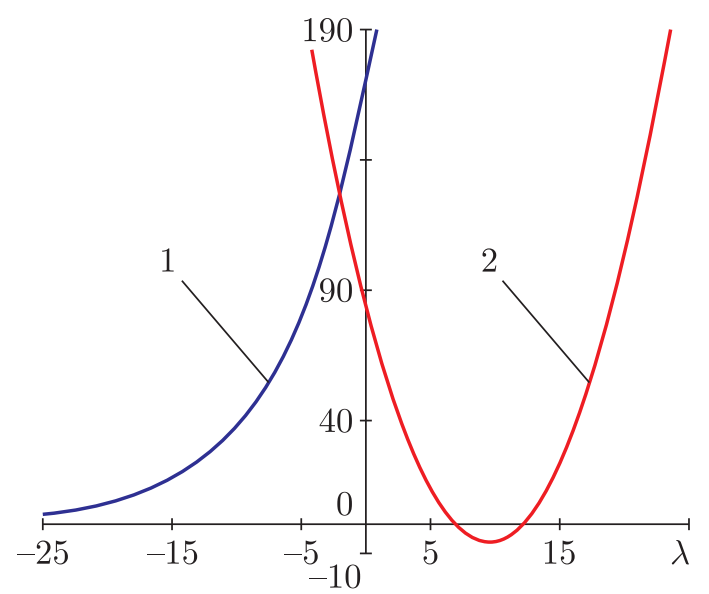

Fig. 7. Solution of the set of equations (3.5) for $\psi_{1}=0.3, \psi_{2}=0.12, \phi_{1}=0.85, \phi_{2}=0.3, w=0.3$ : 1 - the dependence $\left(\lambda^{2}-a \lambda+b\right) ; 2$ - the dependence $d(1+4 w) 2 e^{\lambda w / 2}$.

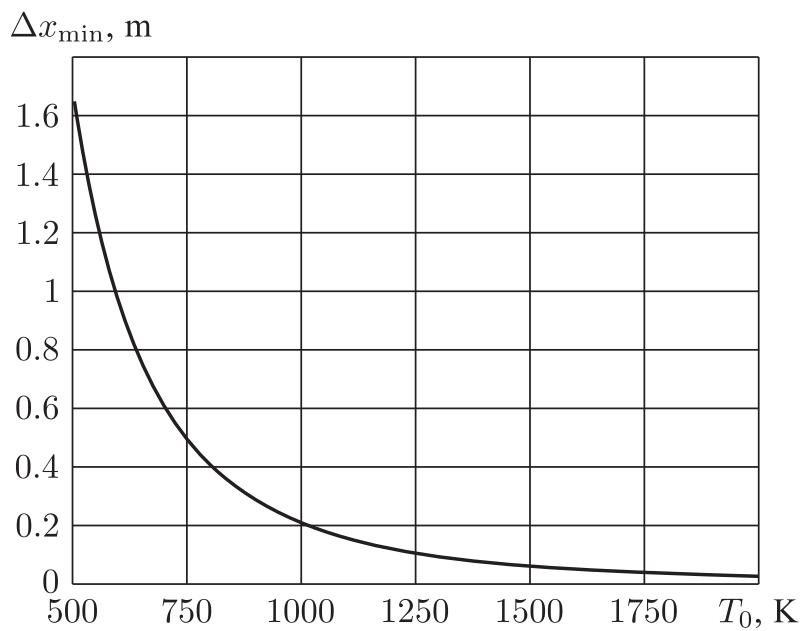

Fig. 8. The dependence $\Delta x_{\min }\left(T_{0}\right) . \psi_{1}=0.3, \psi_{2}=0.12, \phi_{1}=0.85, \phi_{2}=0.3, w=0.3, r=100 \mu \mathrm{m}$, $\rho=800 \mathrm{~kg} / \mathrm{m}^{3}, c=800 \mathrm{Jkg} / \mathrm{K}, u=2 \mathrm{~m} / \mathrm{s}$.

that the relation (3.6) is applicable in the case where $x>\Delta x$, it was assumed that $\Delta x=x$. For the droplet flow parameters discussed above the solution of the equation

$$
w_{b}=\alpha T_{0}^{3} \Delta x\left(1+3 \alpha T_{0}^{3} \Delta x\right)^{-1}
$$

was found for $w_{b}=0.14$. Figure 8 presents the dependence $\Delta x_{\min }\left(T_{0}\right)$. As the temperature increases, $\Delta x_{\min }$ decreases rapidly. However, at reasonable values of the temperature the magnitude $\Delta x_{\text {min }}$ is large enough and makes up a few tens of centimeters.

It follows from the relation (3.6) that, as the droplet flow propagates, the magnitude $w$ decreases fast and becomes less than $w_{b}$, resulting in a decay of the temperature perturbations. On the other hand, in the vicinity of the generator the droplet veil approaches the state of thermodynamic equilibrium, and the radiation cooling process is not accompanied by development of any instabilities. Thus, the instability of the droplet flow radiation cooling process can exist in the limited region only. 


\section{The effect of long-range overradiations on droplets cooling}

The numerical solution of the system (3.1) was found for: $\Psi_{p}=0.15, \Psi_{c}=0, \Delta x / l=0.05$, $\psi_{1}=0.3, \psi_{2}=0.12, \varphi_{1}=0.85, \varphi_{2}=0.3, \varepsilon=1, \alpha T_{0}^{3} l=7 / 3$. The finite value of $\xi$ in the calculations is equal to 0.4 . The initial value is $\tau_{p}=\tau_{c}=1$.

The calculation results are presented in Fig. 9. It is seen that the system of equations at hand has oscillation solutions. In some cases the oscillations may have rather a large amplitude. We point out that the trajectory 2 corresponding to the solution of the idealized problem without regard for external radiation and far radiation interactions terminates at distances well away from the equilibrium position $\left(\tau_{c}^{*} \approx 2.36, \tau_{p}^{*} \approx 1.67\right)$.

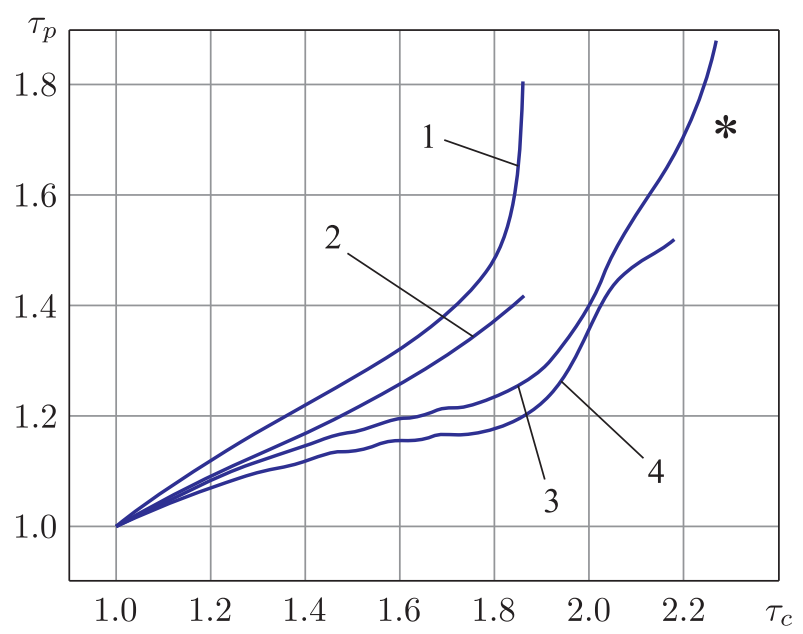

Fig. 9. The numerical solution of the system of Eqs. (3.1). 1 - solar radiation allowed for; 2 without regard for solar radiation and far radiation interactions; 3 - solar radiation and far radiation interactions allowed for; 4 - far radiation interactions allowed for; the equilibrium position of the idealized problem (2.4).

Under the assumption that the droplets mass in the core of the droplet flow is equal to the mass of droplets at its periphery, the weight-average temperature of the flow, $T_{c}$, may be represented at the point with the coordinate $x$ in the form

$$
T_{c}(x)=T_{0} \xi^{4}(x)\left(\tau_{c}(x)+\tau_{p}(x)\right) / 2 .
$$

It follows from the relation (4.1) that the influence of the process of establishing the temperature profile on the weight-average temperature of the flow and the DR thermal power is described by the dependence on the coordinate of the complex $\tau_{c p}=\left(\tau_{c}+\tau_{p}\right) / 2$. The oscillation amplitude of this complex for trajectories of the system, shown in Fig. 9, may attain as high as $5 \%$ of the LDR power. The rate of the oscillations damping is consistent with the analytical results obtained in Section 3.

\section{The numerical solution of the droplet cooling flow equations}

The numerical solution of the system of integro-differential equations (1.3) was obtained for the droplet veil involving the seven droplet layers and having the shape of a rectangular parallelepiped. When carrying out the first calculation, the flow thickness was assumed to be $0.1 \mathrm{~m}$; 


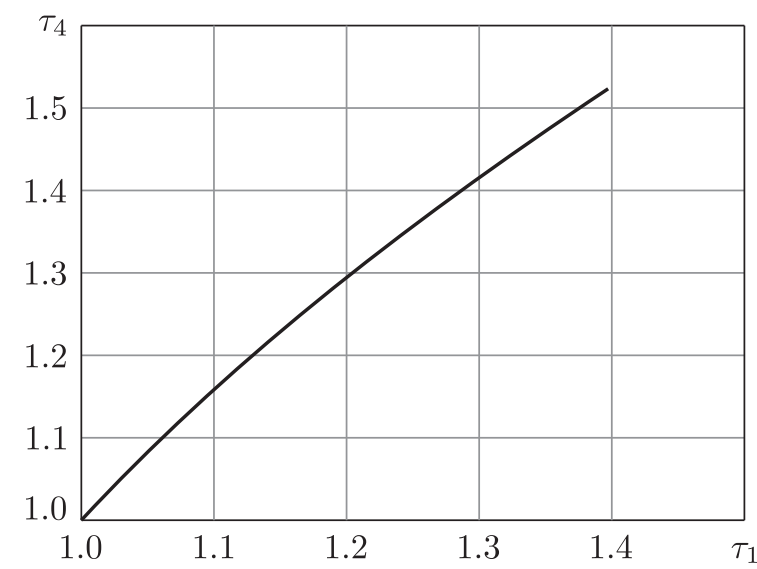

(a)

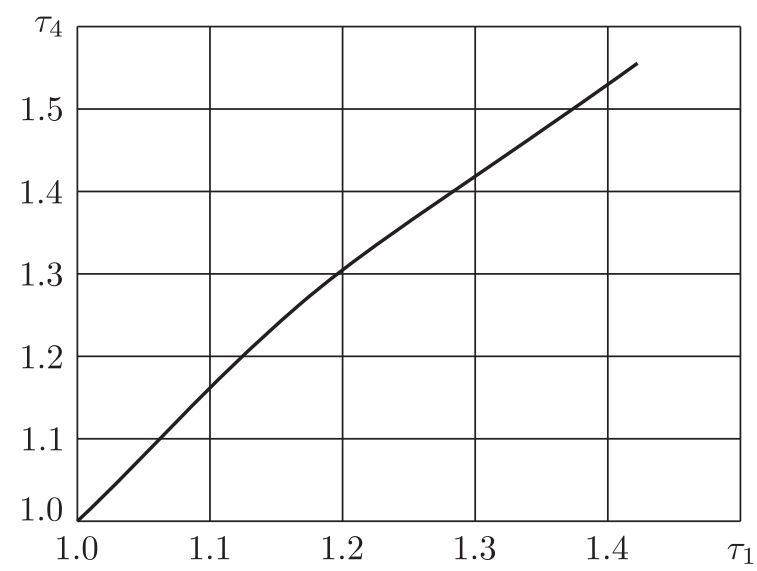

(b)

Fig. 10. The trajectory of the system (1.3) for different thicknesses of the flow: a) $0.1 \mathrm{~m}$ and b) $0.5 \mathrm{~m}$.

for the second calculation, $0.5 \mathrm{~m}$. The length of the droplet flight was equal to $l=10 \mathrm{~m}$, the radius $r=50 \mu \mathrm{m}$, the initial temperature $T_{0}=600 \mathrm{~K}$, the working fluid density $\rho=1100 \mathrm{~kg} / \mathrm{m}^{3}$, $c=600 \mathrm{~J} /(\mathrm{kg} \cdot \mathrm{K})$. Packing of particles in the droplet layers was thought to be square, the spacing between neighboring particles was $250 \mu \mathrm{m}$. function

The function $f_{|i-j|}(x)$ was calculated using the method set forth in [6]. Figure 8 shows the

$$
F_{1}(x)=\frac{1}{\varphi_{1}} \int_{-\infty}^{x} f_{1}(\widetilde{x}) d \widetilde{x}
$$

subject to the coordinate, expressed in units of $h$ which is the distance between the droplet at hand and the droplet layer. It is seen from the plot that the characteristic length of radiation interactions is $\sim 10 \mathrm{~h}$. Thus, for the $0.1 \mathrm{~m}$ thick droplet flow it is necessary to take into account the radiation interactions between the droplets spaced $\sim 1 \mathrm{~m}$ apart, $0.5 \mathrm{~m}$ in thickness, at a distance of $\sim 5 \mathrm{~m}$.

Figure 10 depicts the phase trajectory of the droplet flow cooling process in the coordinates $\left(\tau_{1} ; \tau_{4}\right)$, where $\tau_{1}$ is the dimensionless temperature of the boundary droplet layer numbered 1 , and $\tau_{4}$ is the dimensionless temperature of the central droplet layer numbered 4 (the arrangement of the droplet layers is shown in Fig. 3) for flow thicknesses of 0.1 and $0.5 \mathrm{~m}$. With increasing range of the radiation interactions of particles, the oscillation phenomena accompanying the flow cooling enhance their intensity.

\section{Conclusion}

The effect of far radiation interactions on the development of heat waves in the radiation cooling disperse flow of the low-grade LDR has been numerically and analytically investigated. It has been revealed that the droplets radiation cooling and the process of establishing the equilibrium profile in the flow may be accompanied by oscillation processes. The intensity of oscillation phenomena was designed, and the rate of their damping was determined. The results of the theoretical analysis and the numerical calculation of the actual droplet flow were compared. The effect of long-range radiation interactions on the LDR heat power was determined. 


\section{References}

[1] Demyanko, Yu. G., Konyukhov, G. V., Koroteev, A.S., Kuzmin, E.P., and Pavel'ev, A. A., Nuclear Rocket Engines, Moscow: Norma-Inform, 2001 (Russian).

[2] Siegel, R., Separation of Variables Solution for Nonlinear Radiative Cooling, Int. J. Heat Mass Transf., 1987, vol. 30, no. 5, pp. 959-965.

[3] Dombrovsky, L. A., Radiation Heat Transfer in Disperse Systems, New York: Begell House, 1996.

[4] Ohta, K. O., Graf, R. T., and Ishida, H., Evaluation of Space Radiator Performance by Simulation of Infrared Emission, Appl. Spectrosc., 1988, vol. 42, no. 1, pp. 114-120.

[5] Safronov, A. A., Features of Capillary Breakup of a Liquid Jet at Ohnesorge Numbers Larger Than Unity, J. Eng. Phys. Thermophys., 2017, vol.90, no.1, pp.167-175; see also: Inzh.-Fiz. Zh., 2017, vol. 90, no. 1, pp. 176-185.

[6] Koroteev, A. A., Safronov, A.A., and Filatov, N. I., Influence of the Structure of a Droplet Sheet on the Capacity of Frameless Space Radiators and the Efficiency of the Power Units, High Temp., 2016, vol. 54, no. 5, pp. 767-770; see also: Teplofiz. Vys. Temp., 2016, vol. 54, no. 5, pp. 817-820.

[7] Zel'dovich, Ya. B. and Raizer, Yu. P., Physics of Shock Waves and High-Temperature Hydrodynamic Phenomena, New York: Dover, 2002.

[8] Safronov, A.A., Filatov, N. I., Koroteev, A.A., and Bondareva, N.V., Temperature Stabilization in Dispersed Flows of Frameless Heat Removal Systems in Space, Thermophys. Aeromech., 2017, vol. 24, no. 6, pp. 957-960. 